\title{
Robust Control of a 3D Space Robot with an Initial Angular Momentum based on the Nonlinear Model Predictive Control Method
}

\author{
Tatsuya Kai \\ Department of Applied Electronics \\ Faculty of Industrial Science and Technology \\ Tokyo University of Science \\ 6-3-1 Niijuku, Katsushika-ku, Tokyo 125-8585 JAPAN
}

\begin{abstract}
This paper considers robust control problems for a 3D space robot of two rigid bodies connected by a universal joint with an initial angular momentum. It is particularly difficult to measure an initial angular momentum in parameters of space robots since the value of an initial angular momentum depends on the situations. Hence, the main purpose of this paper is to develop a robust controller with respect to initial angular momenta for the 3D space robot. First, a mathematical model, some characteristics, and two types of control problems for the 3D space robot are presented. Next, for the robust attitude stabilization control problem of the 3D space robot, a numerical simulation is performed by using the nonlinear model predictive control method. Then, for the robust trajectory tracking control problem of the 3D space robot, another numerical simulation is carried out. As a result, it turns out that this approach can realize robust control on initial angular momenta for the two control problems. In addition, computation amount is reduced by this approach and real-time control of the $3 \mathrm{D}$ space robot can be achieved.
\end{abstract}

Keywords-3D space robot; universal joint; initial angular momentum; nonlinear model predictive control; robustness; attitude stabilization control; trajectory tracking control

\section{INTRODUCTION}

Since early times, "the falling cat phenomenon" is well known and this means that a cat can land on her feet despite it drops upside down from a tree. This phenomenon has been focused and investigated in the field of classical mechanics [1]. Similar examples of the falling cat phenomenon can be easily found in the research field of engineering; robots and craft in outer space, freely falling multi-body systems [2], and gymnastic robots [3]. In such systems, the total angular momentum is conserved, and then the conservation law of the total angular momentum can be regarded as a nonholonomic constraint [4]. The word "nonholonomic" means that constraints in the differential equation form are nonintegrable. Therefore, it is possible to change the attitude of the system by changing its shape due to the existence of the nonholonomic constraint.

In the research field of control theory, various researches on space robots have been done so far. In [1], some interesting results on the falling cat phenomenon are shown. In [5], a space robot model of two rigid bodies is dealt with and a near-optimal control law using finite dimensional Fourier basis is developed. In [6], a chained-form based control strategy is proposed to control the attitude of a planer space robot. In [7], a control method by the genetic algorithm for a space robot is derived. In most researches about control of space robots, it is assumed that space robots do not have initial angular momenta. However, space robots often have initial angular momenta in realistic situations. For example, a mother ship gives a space robot out, the space robot obtains an initial angular momentum. Thus, the authors have focused on $3 \mathrm{D}$ space robots with initial angular momenta and developed a control strategy based on the near-optimal control method [8][10]. In these studies, it is confirmed that the proposed method can make the state of the 3D robot transfer to a desired one at a desired time (the state transition control problem). Moreover, in order to deal with other control purposes except the state transition control problem, The authors apply the nonlinear model predictive control method, which is one of the feedback controllers, to 3D space robots [11], [12]. In [11], [12], the two kinds of control problems, called the attitude stabilization control problem and the trajectory tracking control problem, are considered. In these work, it is assumed that initial angular momenta can be measured accurately. However, as compared to physical quantities such as mass, length, and inertia moment, it is quite difficult to measure initial angular momenta because the values of initial angular momenta vary according to the situations. So, when we measure an initial angular momentum of a 3D space robot, there exists error of measurement. To control 3D space robots with initial angular momenta, we have to overcome this problem, that is to say, we have to design robust controllers for initial angular momenta.

The main aim of this research is to construct robust controllers in terms of physical parameter errors for two types of control problems of a 3D space robot with an initial angular momentum, and verify robustness of the new control methods via numerical simulations. The contents of this paper are as follows. First, in Section II, a mathematical model of the 3D universal joint space robot with an initial angular momentum and its some characteristics are presented. Next, in Section III, a numerical simulation on robust attitude stabilization control of the 3D space robot is carried out. Then, Section IV illustrates a numerical simulation on robust trajectory tracking control of the 3D space robot. Finally, Section V gives the concluding remarks of this research and future work. 


\section{3D Universal Joint SPACE Robot With AN INITIAL ANGULAR MOMENTUM}

\section{A. Mathematical Model of 3D Space Robot with I.A.M.}

First, this subsection explains a mathematical model of a 3D universal joint space robot with an initial angular momentum. See [8]-[11] for more details. We consider a space robot that consists of two rigid bodies in the 3-dimensional space as illustrated in Fig. 1. In the space robot, two rigid bodies (Rigid Body 1 and 2) are connected by a universal joint via two links (Link 1 and 2). We denote coordinates of the inertial space, Rigid Body 1 and 2 by $C_{0}, C_{1}$ and $C_{2}$, and assume that the origins of $C_{1}$ and $C_{2}$ correspond to the centroids of Rigid Body 1 and 2, respectively.

Let $A_{i} \in S O(3)$ be the attitude of Rigid Body $i(i=1,2)$ with respective to the inertial space $C_{0}$, and $w_{i} \in \mathbf{R}^{3}$ be the angular velocity of Rigid Body $i$. Note that $\hat{w}_{i}=A_{i}^{\top} \dot{A}_{i}$ holds. We use the following notations: $m_{i}$ : the mass of Rigid Body $i\left(\epsilon=m_{1} m_{2} /\left(m_{1}+m_{2}\right)\right), l_{i}$ : the length of Link $i, s_{i}=$ $\left[\begin{array}{lll}0 & 0 & -l_{i}\end{array}\right]^{\top} \in \mathbf{R}^{3}$ : the vector showing the position of the joint w.r.t. $C_{0}, I_{i} \in \mathbf{R}^{3}$ : The inertia tensor of Rigid Body $i$ $\left(J_{i}=I_{i}+\epsilon \hat{s}_{i}^{\top} \hat{s}_{i}, J_{12}=\epsilon \hat{s}_{1}^{\top} A_{1}^{\top} A_{2} \hat{s}_{2}\right)$, where ${ }^{\wedge}$ is the operator that transforms a 3-dimensional vector $v=\left[\begin{array}{lll}v_{1} & v_{2} & v_{3}\end{array}\right]^{\top} \in \mathbf{R}^{3}$ into a $3 \times 3$ skew-symmetric matrix:

$$
\hat{v}=\left[\begin{array}{ccc}
0 & -v_{3} & v_{2} \\
v_{3} & 0 & -v_{1} \\
-v_{2} & v_{1} & 0
\end{array}\right]
$$

It is also noted that $A:=A_{1}^{\top} A_{2}$ represents the shape of the space robot and

$$
w_{2}=A^{\top} w_{1}+w
$$

holds for the angular velocity of the joint $w \in \mathbf{R}^{3}, \hat{w}=$ $A^{\top} \dot{A}$. In this paper, we adopt the universal joint depicted in Fig. 2 as a joint connecting the two rigid bodies. It must be noted the universal joint can twist and the degree of freedom is 2. Let $\theta_{1} \in \mathbf{R}$ and $\theta_{2} \in \mathbf{R}$ be angles of Link 1 and 2, respectively, and we use the notation: $\theta=\left[\begin{array}{ll}\theta_{1} & \theta_{2}\end{array}\right]^{\top} \in \mathbf{R}^{2}$. By considering coordinates of the space robot, we can show the following:

$$
A=\left[\begin{array}{ccc}
\sin \theta_{1} \sin \theta_{2} & \cos \theta_{1} & -\sin \theta_{1} \cos \theta_{2} \\
\cos \theta_{2} & 0 & \sin \theta_{2} \\
\cos \theta_{1} \sin \theta_{2} & -\sin \theta_{1} & -\cos \theta_{1} \cos \theta_{2}
\end{array}\right] .
$$

In this paper, we consider the case where the universal model has an initial angular momentum, hence we denote the initial angular momentum by $P_{0} \in \mathbf{R}^{3}$. Then, the conservation law of total angular momentum of the space robot is represented by

$$
\left(A_{1} J_{1}+A_{2} J_{12}^{\top}\right) w_{1}+\left(A_{2} J_{2}+A_{1} J_{12}\right) w_{2}=P_{0},
$$

and we can easily confirm that (4) is represented as $A(q)+$ $B(q) \dot{q}=0$ using the generalized coordinate $q$, and thus this is an affine constraint [14]. From the result in [14], it can be checked that (4) is completely nonholonomic. Assume that angular velocities of the universal joint can be controlled, that is, $u_{1}:=\dot{\theta}_{1}, u_{2}:=\dot{\theta}_{2}$. Then, we have

$$
w=\underbrace{\left[\begin{array}{c}
\cos \theta_{2} \\
0 \\
\sin \theta_{2}
\end{array}\right]}_{b_{1}} u_{1}+\underbrace{\left[\begin{array}{l}
0 \\
1 \\
0
\end{array}\right]}_{b_{2}} u_{2} .
$$

Substituting (2) and (5) into (4), we obtain

$$
A_{1} I_{u} w_{1}+A_{1}\left(A J_{2}+J_{12}\right)\left(b_{1} u_{1}+b_{2} u_{2}\right)=P_{0},
$$

where we define the new notation:

$$
I_{u}:=J_{1}+A J_{2} A^{\top}+A J_{12}^{\top}+J_{12} A^{\top} .
$$

To represent the attitudes of Rigid Body 1, we use the Cayley-Rodrigues parameter, and hence the attitude of Rigid Body $1 A_{1}$ is expressed as (8). using the parameter $\alpha=$ $\left[\begin{array}{lll}\alpha_{1} & \alpha_{2} & \alpha_{3}\end{array}\right]^{\top} \in \mathbf{R}^{3}$. The relationship between $w_{1}$ and $\alpha$ is expressed by

$$
w_{1}=U_{1}(\alpha) \dot{\alpha}, \quad U_{1}(\alpha)=\frac{2(I-\hat{\alpha})}{1+\alpha^{\top} \alpha} .
$$

Substituting (9) into (6) and solving for $\dot{\alpha}$, we have

$$
\begin{aligned}
\dot{\alpha}= & U_{1}^{-1} I_{u}^{-1} A_{1}^{\top} P_{0} \\
& \quad-U_{1}^{-1} I_{u}^{-1}\left(A J_{2}+J_{12}\right)\left(b_{1} u_{1}+b_{2} u_{2}\right) .
\end{aligned}
$$

Consequently, we derive the universal joint model with an initial angular momentum (11) with the variables $q:=$ $\left[\begin{array}{ll}\theta^{\top} & \alpha^{\top}\end{array}\right]^{\top} \in \mathbf{R}^{5}, u:=\left[\begin{array}{ll}u_{1} & u_{2}\end{array}\right]^{\top} \in \mathbf{R}^{2}$. Thus, the system (11) is represented as a 5 -state and 2-input nonlinear affine control system.

\section{B. Control Problems for 3D Space Robot with I.A.M.}

Next, some characteristics of the universal joint model with an initial angular momentum (11) are investigated from the viewpoint of nonlinear control theory. If an initial angular momentum exists, that is, $P_{0} \neq 0$, the drift term of the system (11) satisfies $f(q) \neq 0, \forall q$. This means that the system (11) does not have any equilibrium point, that is, the space robot cannot stop and keeps moving. For nonlinear control systems, the concepts "local accessibility" and "local controllability" are quite important in order to investigate the range of movement of the system [13], [14]. The next proposition on local accessibility and local controllability of the system (11) can be obtained [8]-[11].

Proposition 1: The universal joint model with an an initial angular momentum (11) is locally strongly accessible at any point $q=\left[\begin{array}{ll}\theta^{\top} & \alpha^{\top}\end{array}\right]^{\top} \in \mathbf{R}^{5}$. Moreover, if the control input $u$ is sufficiently large, (11) is small-time locally controllable at any point $q$.

Since there is no equilibrium point in the model of the universal joint model with an initial angular momentum (11), we cannot deal with a normal stabilization problem for the system. However, Proposition 1 guarantees that we have some possibilities of other control purposes except normal stabilization. For the universal joint model with an initial angular momentum (11), the next three types of control purposes 


$$
\begin{aligned}
& A_{1}(\alpha)=\frac{1}{1+\|\alpha\|^{2}}\left[\begin{array}{ccc}
1+\alpha_{1}^{2}-\alpha_{2}^{2}-\alpha_{3}^{2} & 2\left(\alpha_{1} \alpha_{2}-\alpha_{3}\right) & 2\left(\alpha_{1} \alpha_{3}+\alpha_{2}\right) \\
2\left(\alpha_{1} \alpha_{2}+\alpha_{3}\right) & 1-\alpha_{1}^{2}+\alpha_{2}^{2}-\alpha_{3}^{2} & 2\left(\alpha_{2} \alpha_{3}-\alpha_{1}\right) \\
2\left(\alpha_{1} \alpha_{3}-\alpha_{2}\right) & 2\left(\alpha_{2} \alpha_{3}+\alpha_{1}\right) & 1-\alpha_{1}^{2}-\alpha_{2}^{2}+\alpha_{3}^{2}
\end{array}\right] \\
& {\left[\begin{array}{c}
\dot{\theta}_{1} \\
\dot{\theta}_{2} \\
\dot{\alpha}
\end{array}\right]=\underbrace{\left[\begin{array}{c}
0 \\
0 \\
U_{1}^{-1} I_{u}^{-1} A_{1}^{\top} P_{0}
\end{array}\right]}_{f(q)}+\underbrace{\left[\begin{array}{cc}
0 \\
0 & 1 \\
-U_{1}^{-1} I_{u}^{-1}\left(A J_{2}+J_{12}\right) b_{1} & -U_{1}^{-1} I_{u}^{-1}\left(A J_{2}+J_{12}\right) b_{2}
\end{array}\right]}_{g(q)}\left[\begin{array}{l}
u_{1} \\
u_{2}
\end{array}\right]}
\end{aligned}
$$

can be considered: (i) the state transition control problem: a controller to transfer of the space robot to a desired state at a desired time; (ii) the attitude stabilization control problem: a controller to stabilize only the attitude of the space robot with ignoring the shape of it; (iii) the trajectory tracking control problem: a controller to track the space robot to a given reference trajectory. In this paper, we will tackle the problems (iii): the trajectory tracking control problem.

\section{Robust Attitude Stabilization Control}

\section{A. Problem Setting}

In this section, we shall deal with "the robust attitude stabilization control problem" for the universal joint model with an initial angular momentum (11). The purpose of this control problem is that we design a controller to stabilize only the attitude of the space robot $\alpha$ with ignoring the shape $\theta$ in the presence of a parameter error for the initial angular momentum. For example, this kind of the control problem includes the situation where we move the space robot to the direction of a given point of the earth in order to send and receive information. The robust attitude stabilization control problem is formulated as the next.

Problem 1 [Robust Attitude Stabilization Control]: For the universal joint model with an initial angular momentum (11), find control inputs such that the attitude of Rigid Body $1 \alpha$ is stabilized to a desired value $\alpha_{d}$ under the assumption on the existence of a parameter error for the initial angular momentum $\left(\hat{P}_{0}\right.$ : an estimated value, $P_{0}:$ a real value).

In this paper, we take the nonlinear model predictive control approach in order to solve Problem 1. Especially, we use the C/GMRES method [15], which is a real-time optimal control algorithm for nonlinear systems. In the simulation, we use the parameters of the 3D space robot: $l_{1}=l_{2}=1, m_{1}=m_{2}=$ $1, I_{1}=I_{2}=\operatorname{diag}\{1 / 2,1 / 2,1\}$, the estimated value of the initial angular momentum: $\hat{P}_{0}=[0.10 .1-0.1]^{\top}$, the real value) of the initial angular momentum: $P_{0}=\left[\begin{array}{lll}0.07 & 0.07-0.01\end{array}\right]^{\top}$, the initial state: $q_{0}=\left[\begin{array}{llll}\pi / 2 & \pi / 2 & 1 & 1\end{array}\right]^{\top}$, the desired attitude: $\alpha_{d}=\left[\begin{array}{lll}0 & 0 & 0\end{array}\right]^{\top}$. For the nonlinear model predictive control method, we use the next cost function:

$$
\begin{aligned}
J= & \frac{1}{2} \int_{t}^{t+T(t)}\left(\alpha(\tau)-\alpha_{d}\right)^{\top} Q\left(\alpha(\tau)-\alpha_{d}\right) d \tau \\
& +\frac{1}{2} \int_{t}^{t+T(t)} u(\tau)^{\top} R u(\tau) d \tau \\
& +\frac{1}{2}\left(\alpha(t+T)-\alpha_{d}\right)^{\top} S\left(\alpha(t+T)-\alpha_{d}\right)
\end{aligned}
$$

with the weight matrices:

$$
\begin{aligned}
& Q=\operatorname{diag}\{2.0,1.0,3.0\}, \\
& R=\operatorname{diag}\{0.01,0.01\}, \\
& S=\operatorname{diag}\{0.8,0.2,0.4\} .
\end{aligned}
$$

Note that the cost function (12) evaluates only $\alpha$ and ignores $\theta$ since we consider attitude stabilization. In (12), the evaluation interval is set as $T(t)=T\left(1-e^{-a t}\right), T=6.5, a=$ 0.05. Moreover, we also use the parameters of controller: the division number of the evaluation interval: $N=50$, the stabilization parameter of the continuation method: $\zeta=20$, the number of iterations of the GMRES method: $k_{\max }=3$, the sampling time: $\Delta t=0.05[\mathrm{~s}]$, the simulation time: $20[\mathrm{~s}]$.

\section{B. Simulation Results}

A numerical simulation is performed based on the problem setting in subsection III-A. Fig. 2 and 3 illustrate the simulation results. Fig. 2 shows the time series of $\theta$ and $\alpha$ of the 3D space robot, and Fig. 3 depicts snapshots of the behavior of the 3D space robot. From these results, we can confirm that the attitude of Rigid Body $1 \alpha$ is stabilized to the desired value $\alpha_{d}=\left[\begin{array}{lll}0 & 0 & 0\end{array}\right]^{\top}$. The computation time of this simulation is $0.95 \mathrm{~s}$, and hence we can see that real-time robust control can be achieved by the proposed method. It is also confirmed that the control purposes can be achieved for other problem settings (physical parameters of the 3D space robot, initial and desired states, and an initial angular momentum) with tuning the weight matrices in (12).

\section{Robust Trajectory TRacking CONTROL}

\section{A. Problem Setting}

Next, in this section, we shall deal with another control purpose, called "the robust trajectory tracking control problem" for the universal joint model with an initial angular momentum (11). The purpose of this control problem is that we design a controller to make the state of space robot $q$ track to a desired trajectory data $q_{d}(t)$ in the presence of a parameter error for the initial angular momentum. Examples of the control problem are the situations where we track a solar panel installed into the space robot to the direction of the sun, and we shoot moving astronomical bodies with a camera installed into the space robot. The robust trajectory tracking control problem is defined as follows.

Problem 2 [Robust Trajectory Tracking Control] : For the universal joint model with an initial angular momentum (11), find control inputs such that the state $q$ tracks to a desired trajectory $q_{d}(t)$ under the assumption on the existence of a parameter error for the initial angular momentum $\left(\hat{P}_{0}\right.$ : an estimated value, $P_{0}$ : a real value). 


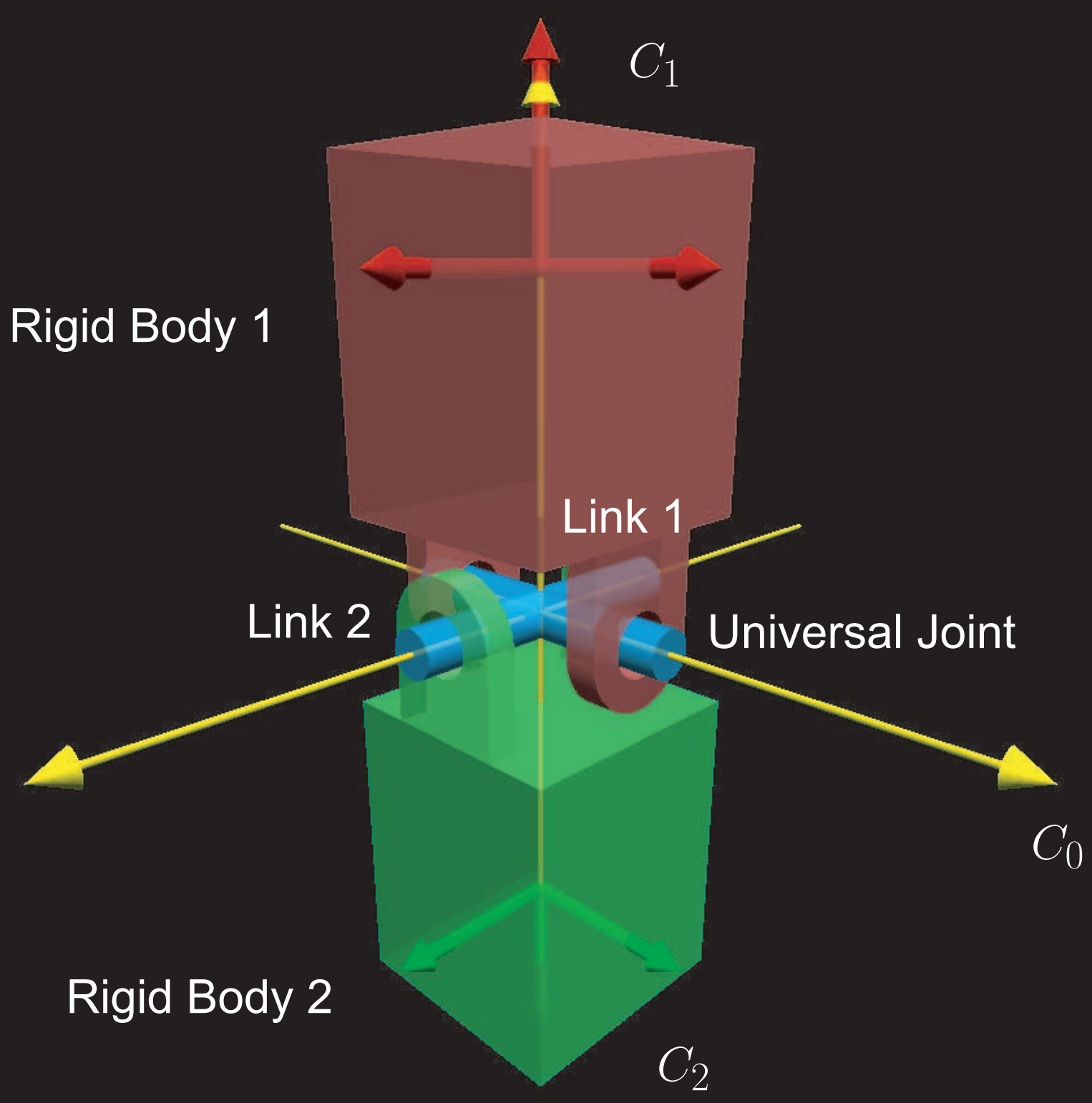

Fig. 1. The 3D universal joint space robot model.

To solve Problem 2, we also utilize the C/GMRES method [15]. In a simulation, we use the parameters of the 3D space robot: $l_{1}=l_{2}=1, m_{1}=m_{2}=1, I_{1}=I_{2}=$ $\operatorname{diag}\{1 / 2,1 / 2,1\}$, the estimated value of the initial angular momentum: $\hat{P}_{0}=\left[\begin{array}{lll}0.1 & -0.1 & 0.2\end{array}\right]^{\top}$, the real value) of the initial angular momentum: $P_{0}=\left[\begin{array}{lll}0.095 & -0.095 & 0.195\end{array}\right]^{\top}$, the initial state: $q_{0}=\left[\begin{array}{lllll}-\pi / 2 & -\pi / 2 & -1 & -1 & -1\end{array}\right]^{\top}$. The desired trajectory $q(t)$ is generated from (11) in advance (see the simulation result in the next subsection). For the C/GMRES method, we use the next cost function:

$$
\begin{aligned}
J= & \frac{1}{2} \int_{t}^{t+T(t)}\left(q(\tau)-q_{d}(\tau)\right)^{\top} Q\left(q(\tau)-q_{d}(\tau)\right) d \tau \\
& +\frac{1}{2} \int_{t}^{t+T(t)} u(\tau)^{\top} R u(\tau) d \tau \\
& +\frac{1}{2}\left(q(t+T)-q_{d}(t)\right)^{\top} S\left(q(t+T)-q_{d}(t)\right),
\end{aligned}
$$



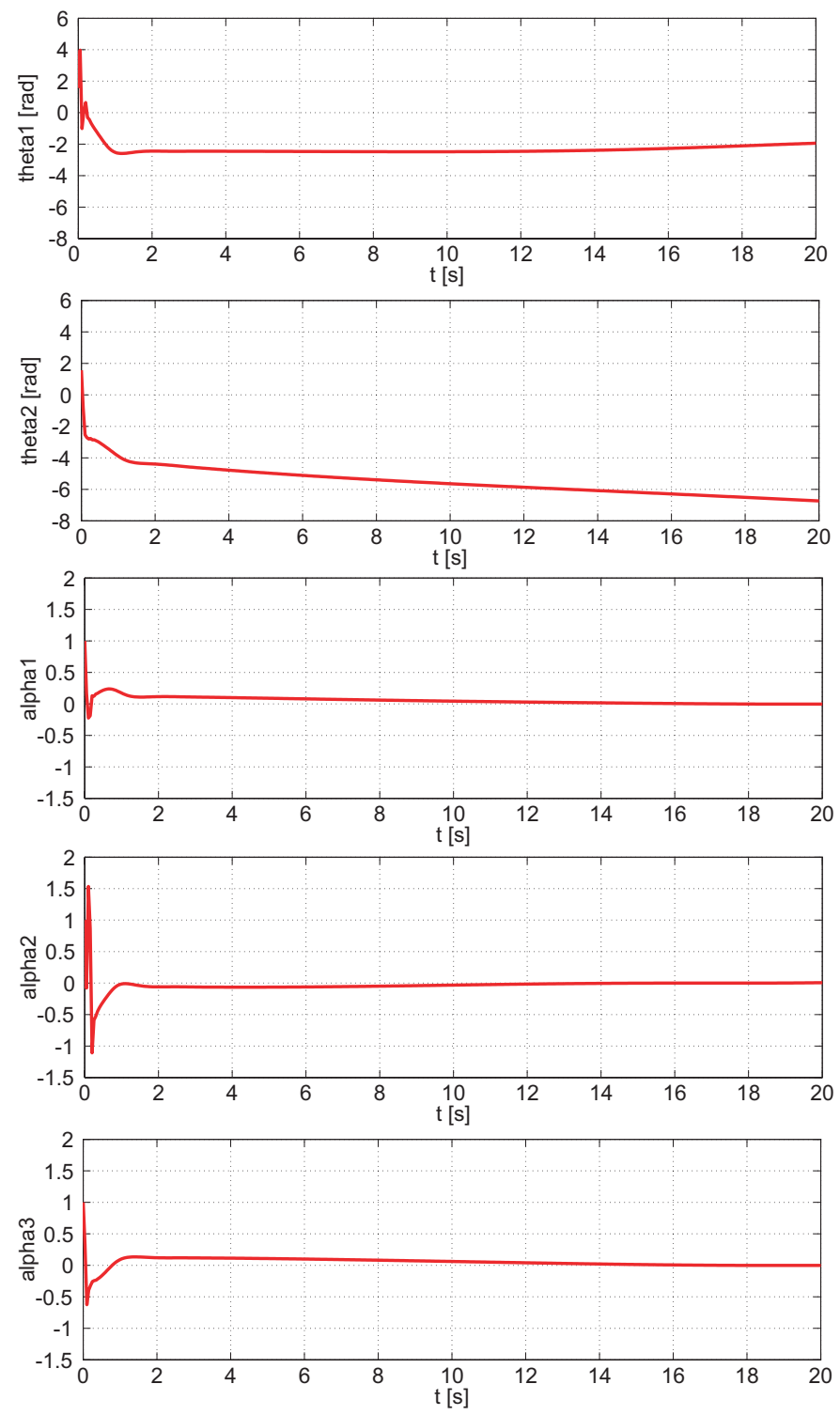

Fig. 2. The simulation result in the robust attitude stabilization control problem: the time histories of the real trajectory.

where

$$
\begin{aligned}
& Q=\operatorname{diag}\{0.02,0.015,0.04,0.04,0.04\} \\
& R=\operatorname{diag}\{0.01,0.01\} \\
& S=\operatorname{diag}\{0.1,0.1,0.2,0.2,0.2\}
\end{aligned}
$$

and the evaluation interval $T(t)=T\left(1-e^{-a t}\right), T=$ $6.5, a=0.05$. In addition, we also use the parameters of controller: the division number of the evaluation interval: $N=50$, the stabilization parameter of the continuation method: $\zeta=200$, the number of iterations of the GMRES method: $k_{\max }=3$, the sampling time: $\Delta t=0.005[\mathrm{~s}]$, the simulation time: $40[\mathrm{~s}]$.

\section{B. Simulation Results}

The simulation results are depicted in Fig. 4, 5, and 6. Fig. 4 shows both the time series of $\theta, \alpha$ of the space robot and the desired trajectory. In Fig. 5, the time series of the error defined by $e(t):=q(t)-q_{d}(t)$ is illustrated. In addition, snapshots of the behavior of the 3D space robot for both desired trajectory and the real one are shown in Fig. 6. From these results, we can see that the state of the space robot tracks to the desired trajectory $q_{d}(t)$ and then the error $e(t)$ converges to 0 . The computation time of this simulation is $2.71 \mathrm{~s}$, and hence it turns out that real-time robust control can be achieved by the proposed method. We can also confirm that the control purposes can be also achieved for other problem settings (physical parameters of the 3D space robot, initial and desired states, an initial angular momentum, and a desired trajectory) with tuning the weight matrices in (12).

\section{CONCLUSIONS}

This paper has considered two kinds of robust control problems "the robust attitude stabilization control problem" and "the robust trajectory tracking control problem" for the $3 \mathrm{D}$ universal joint space robot with an initial angular momentum 


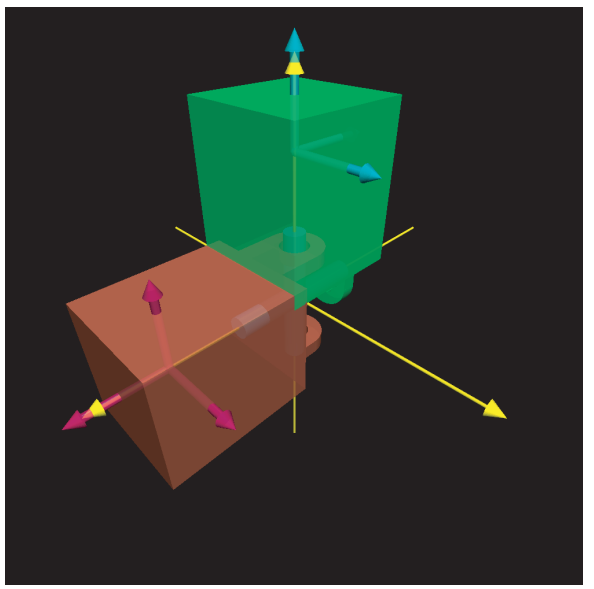

(a) $t=0.0000$

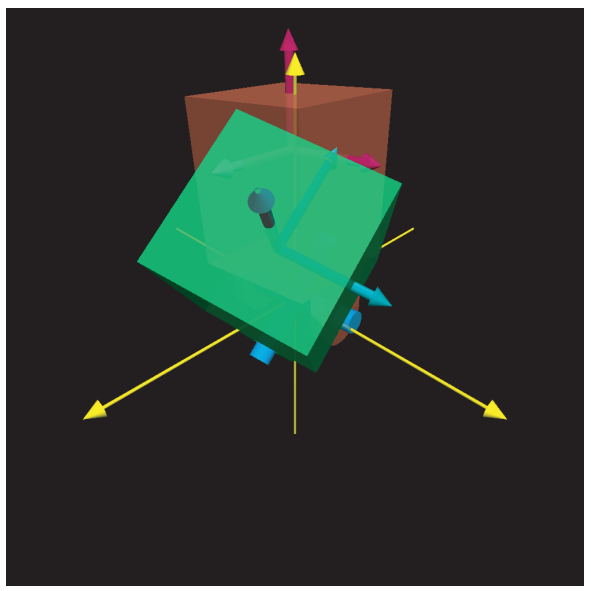

(d) $t=12.0000$

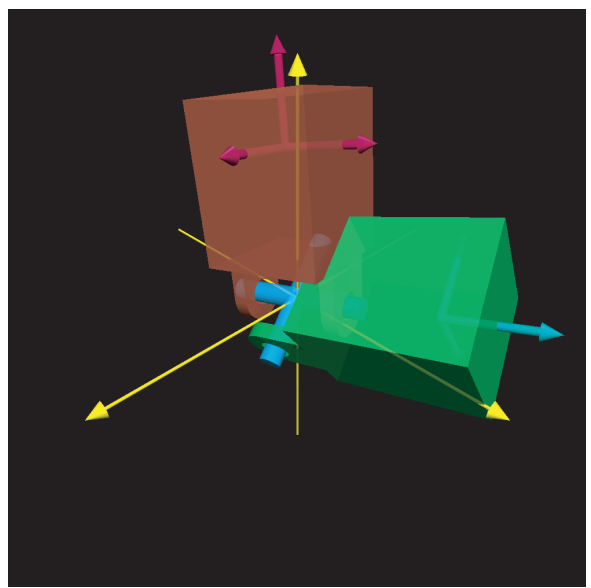

(b) $t=4.0000$

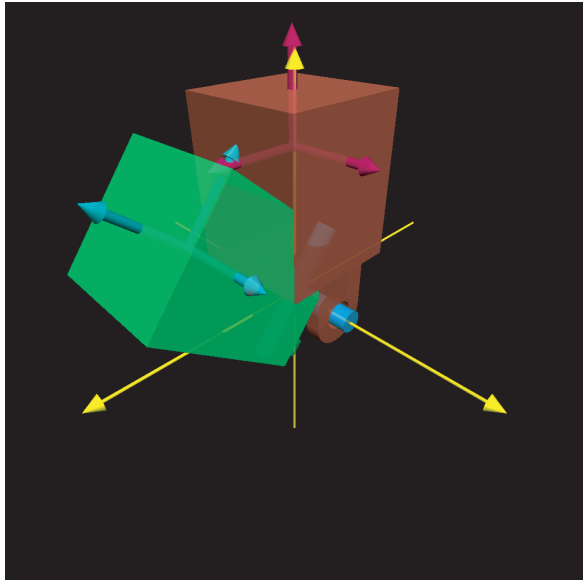

(e) $t=16.0000$

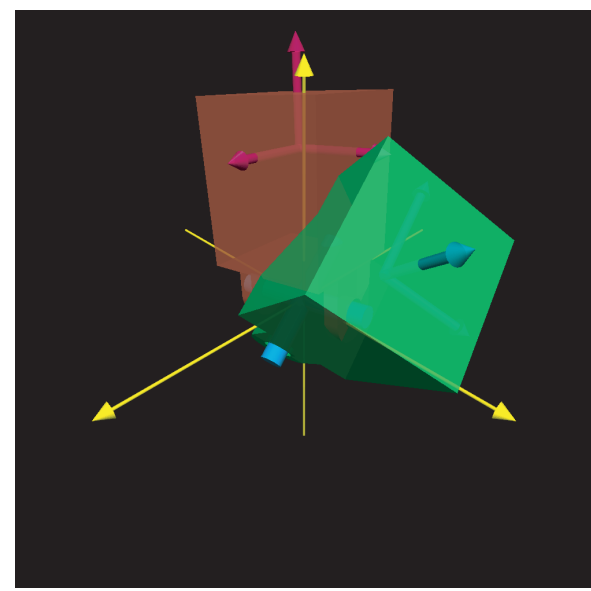

(c) $t=8.0000$

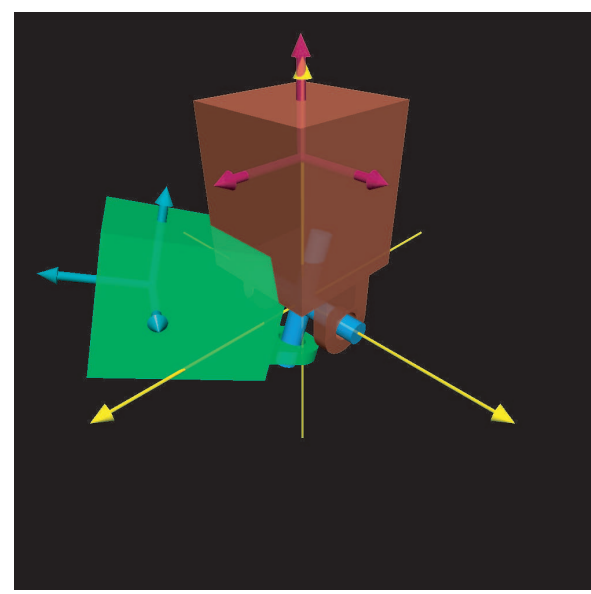

(f) $t=20.0000$

Fig. 3. The simulation result in the robust attitude stabilization control problem: the snapshots of the 3D space robot.

from the standpoint of the nonlinear model predictive control approach. The simulation results have shown that the control purposes are achieved in both robust control problems, and hence the proposed method has robustness for initial angular momenta. Moreover, we can see that the computation times in numerical simulations are quite short, and thus real-time control has been realized.

Our future work include the next topics: modeling and control of space robots with initial angular momenta by the quaternion representation, controller design for space robot models in the descriptor representation, and control of other types of $3 \mathrm{D}$ space robots.

\section{REFERENCES}

[1] M. J. Enos, ed., "Dynamics and control of mechanical systems: the falling cat and related problems", Fields Institute Communications, 1, American Mathematical Society, 1993

[2] T. Mita, S. Hyon, and T. Nam, "Analytical Time Optimal Control Solution for Free Flying Objects with Drift Terms", in Proc. of IEEE CDC 2000, Sydney, Australia, pp. 91-94, 2000

[3] T. Mita, S. Hyon and T. Nam, "Analytical Time Optimal Control Solution for a Two Link Planar Acrobot with Initial Angular Momentum", IEEE Trans. Robotics and Automation, Vol. 17, No. 3, pp. 361-366 2001
[4] A. M. Bloch, Nonholonomic Mechanics and Control, Springer, 2003

[5] C. Fernandes, L. Gurvits, and Z. Li, "Near-Optimal Nonholonomic Motion Planning for a System of Coupled Rigid Bodies", IEEE Trans. on Automatic Control, Vol. 39, No. 3, pp. 450-463, 1994

[6] F. Matsuno and J. Tsurusaki, "Chained Form Transformation Algorithm for a Class of 3-States and 2-Inputs Nonholonomic Systems and Attitude Control of a Space Robot", in Proc. of IEEE CDC 1999, Arizona, USA, pp. 2126-2131, 1999

[7] X. Ge and L. Chen, "Attitude Control of a Rigid Spacecraft with Two Momentum Wheel Actuators Uging Genetic Algorithm", Acta Astronautica, Vol. 55, No. 1, pp. 3-8, 2004

[8] T. Kai and K. Tamaki, "3D Attitude Near-Optimal Control of a Universal Joint Space Robot Model with Initial Angular Momentum," in Proc. of ICCA 2009, Christchurch, New Zealand, pp.2335-2340, 2009

[9] T. Kai and K. Tamaki, "Control of a 3D Ball-in-Socket Joint Space Robot Model with Initial Angular Momentum based on the NearOptimal Control Algorithm," in Proc. of NOLCOS 2010, Bologna, Italy, pp. 957-962, 2011

[10] T. Kai and K. Tamaki, "A Near-Optimal Control Approach to 3D Ballin-Socket Joint Space Robot Models with Initial Angular Momenta," Acta Astronautica, Vol. 68, pp. 1702-1711, 2011

[11] T. Kai, "A Model Predictive Control Approach to Attitude Stabilization and Trajectory Tracking Control of a 3D Universal Joint Space Robot with an Initial Angular Momentum," in Proc. of IEEE CDC 2011, Orlando, USA, pp. 3547-3552, 2011 

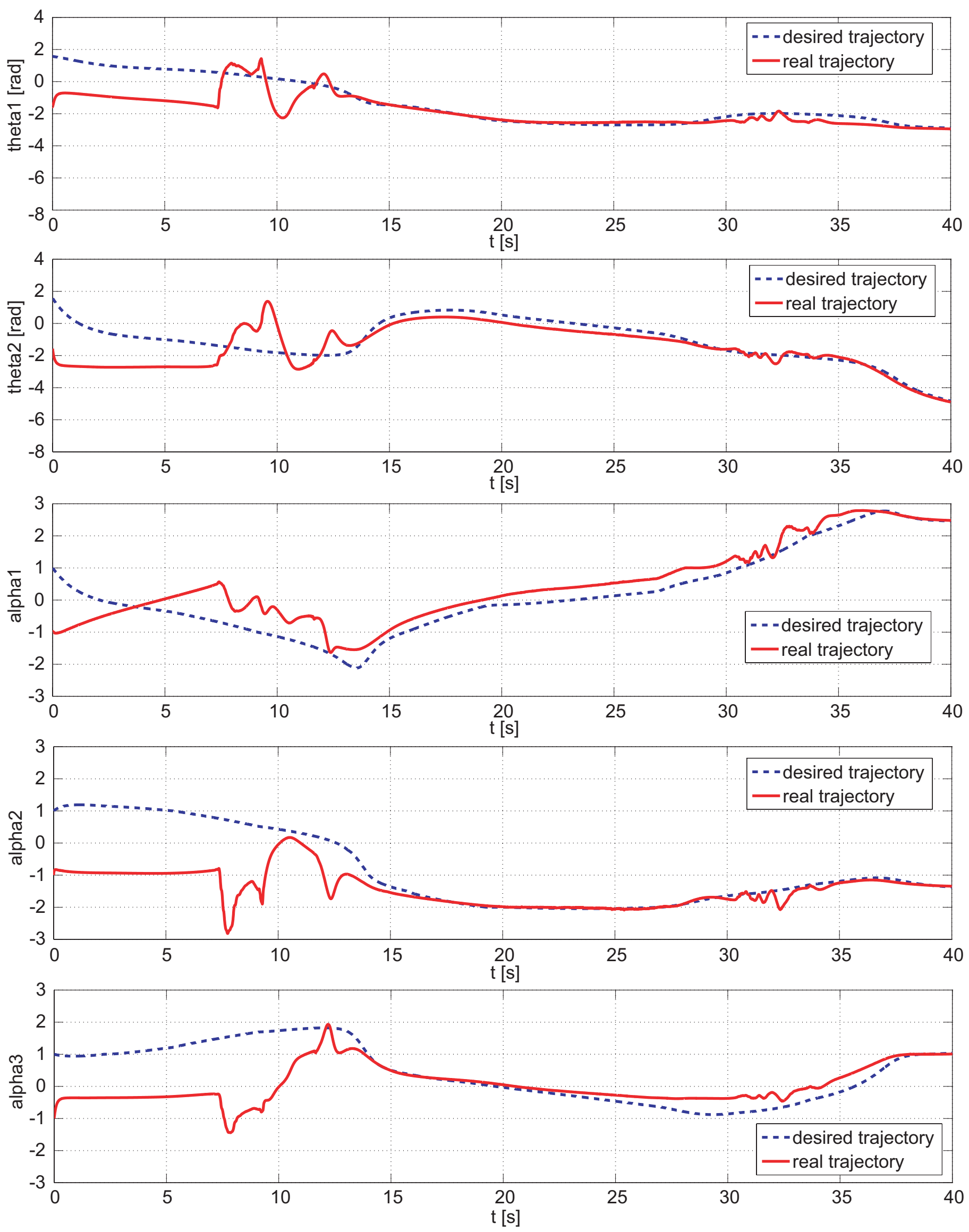

Fig. 4. The simulation result in the robust trajectory tracking control problem: the time histories of the desired and real trajectories. 

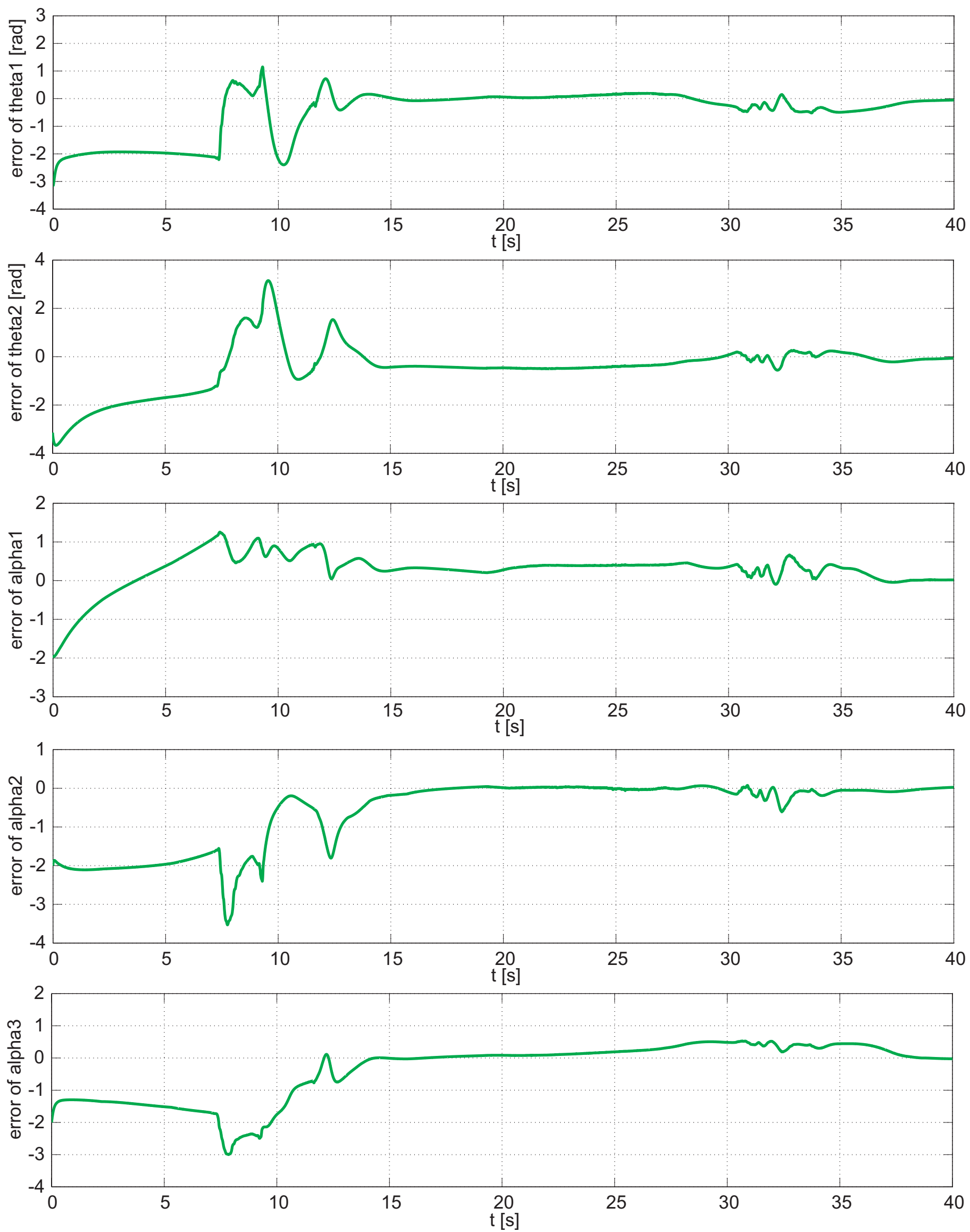

Fig. 5. The simulation result in the robust trajectory tracking control problem: the time histories of the error. 

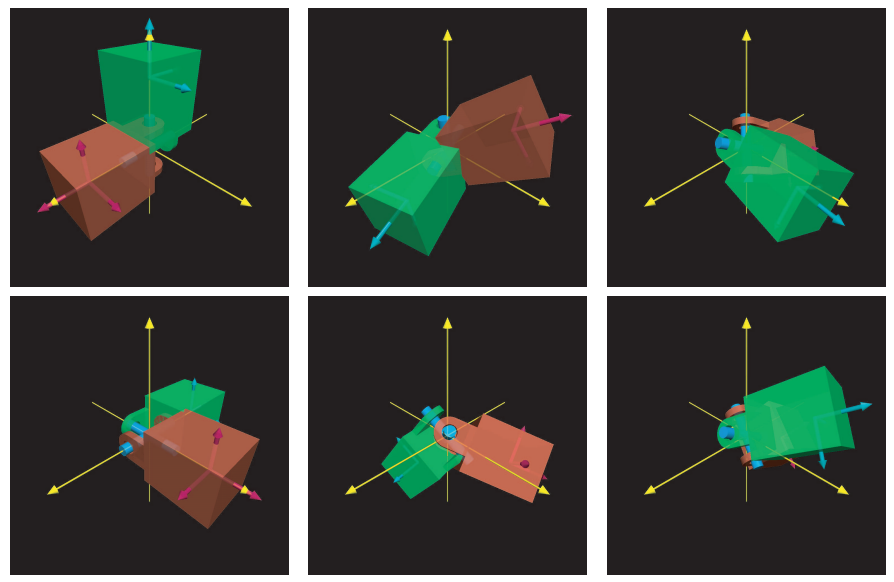

(a) $t=0.0000$

(b) $\mathrm{t}=8.0000$

(c) $\mathrm{t}=16.0000$
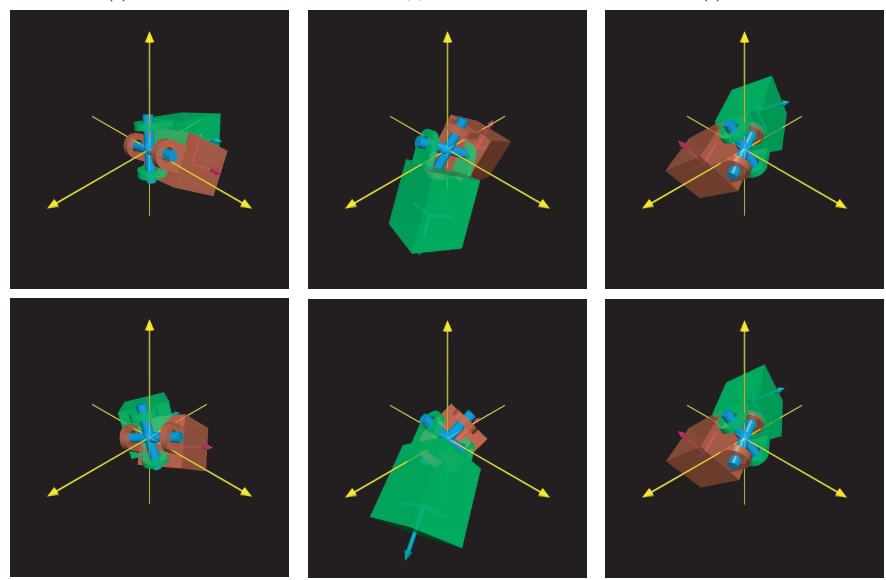

(d) $t=24.0000$

(e) $\mathrm{t}=32.0000$

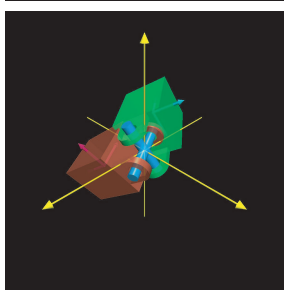

(f) $\mathrm{t}=40.0000$

Fig. 6. The simulation result in the robust trajectory tracking control problem: the snapshot of the 3D space robot (upper: the desired trajectory, lower: the real trajectory).

[12] T. Kai, "Real-time Control of a 3D Space Robot with an Initial Angular Momentum", International Journal of Engineering Research and Industrial Applications, Vol. 7, No. 3, pp. 137-151, 2014

[13] J. M. Godhavn, A. Balluchi, L. S. Crawford, and S. S. Sastry, "Steering of a Class of Nonholonomic Systems with Drift Terms", Automatica, Vol. 35, No. 5, pp. 837-847, 1999

[14] T. Kai, H. Kimura, and S. Hara, "Nonlinear Control Analysis on Kine- matically Asymmetrically Affine Control Systems with Nonholonomic Affine Constraints", Proc. of 16th IFAC World Congress, Seville, Spain, Paper No. Mo-M08-TO/5, 2005

[15] T. Ohtsuka, "A Continuation/GMRES Method for Fast Computation of Nonlinear Receding Horizon Control", Automatica, Vol. 40, No. 4, pp. 563-574, 2004 